\title{
Involving Family and Social Support Systems in Tobacco Cessation Treatment for Patients With Cancer
}

\author{
The Cancer Center Cessation Initiative Family and Social Support Systems Working Group
}

\begin{abstract}
Individuals from the family and social support network of patients with cancer can have a pivotal role in reinforcing patients' efforts to become and remain tobacco-free. This support is critical along the entire continuum of cancer care. Although $\mathrm{NCl}$-designated Cancer Centers across the United States are increasingly offering tobacco cessation services as a result of the $\mathrm{NCl}$ Cancer Center Cessation Initiative (C3I), engaging patients' family and other support network in tobacco treatment is not yet a routine practice. To facilitate the consideration and involvement of patients' social support systems (including family, peers, and non-healthcare provider caregivers), we formed the C3I Family and Social Support Systems Working Group. This paper describes the current practices and challenges among C3I cancer centers in engaging the support systems of patients with cancer in order to reduce tobacco use and/or secondhand smoke exposure. Building on this knowledge, this Working Group proposes a research agenda to facilitate support persons' involvement in tobacco treatment as part of oncology care. The research priorities identified include establishing (1) evidence-based strategies for engaging family and social support systems in patients' cessation efforts, (2) interventions to provide cessation treatment options to support persons, and (3) best practices to routinely identify and engage family and social support systems in patients' cessation efforts.
\end{abstract}

J Natl Compr Canc Netw 2021;19(Suppl 1):S8-11 doi: $10.6004 /$ jnccn.2021.7090

\section{Engaging Family and Social Support Systems in Tobacco Cessation in Cancer Care}

A cancer diagnosis and treatment are stressful life events, both physically and mentally. Social support provided by informal caregivers, family, and friends is correlated with positive treatment outcomes for cancer, significantly reducing psychological distress and improving emotional well-being. ${ }^{1,2}$ Leveraging individuals within a patient's social network, particularly informal caregivers who are living with the patient, can also be helpful in supporting patients with tobacco cessation. ${ }^{3}$ Patients with cancer who use tobacco, or recently stopped tobacco use, benefit from receiving sustained social support in quitting and remaining abstinent. ${ }^{4-6}$ The application of a family systems approach to treat tobacco use among patients with cancer has shown promise. ${ }^{4}$ Mounting literature suggests that secondhand smoke exposure and/or spousal smoking were strong predictors of relapse to smoking among patients with cancer. ${ }^{7-10}$ Thus, helping caregivers and family members to quit smoking is a crucial component of a tobacco treatment program. Furthermore, the benefits of smoking cessation among patients with cancer go beyond the effects on their own cancer recovery and

*A complete list of the collaborators in the Cancer Center Cessation Initiative Family and Social Support Systems Working Group appears at the end of this article. improvement of their quality of life to include creating a tobacco-free living environment for their caregivers and support persons by reducing their exposure to secondhand smoke. Patients with cancer may also serve as a strong motivator for those who smoke to consider smoking cessation. ${ }^{11}$

Although cancer centers across the United States are increasingly offering tobacco cessation services as a result of the NCI Cancer Center Cessation Initiative (C3I), ${ }^{12}$ extending services to those who use tobacco products within patients' social networks is less common. In response, in the Fall of 2020, the C3I Coordinating Center and participating clinicians, researchers, and staff of C3I formed the Family and Social Support Systems Working Group (hereafter referred as the "Working Group"). The mission of the Working Group is to facilitate the involvement of patients' family and social support systems or support persons (including peers, family, and non-healthcare provider caregivers) in tobacco cessation and sustained abstinence of patients with cancer as a pivotal component of all phases of cancer care and survivorship. The primary focus of this manuscript is smoking and smoking cessation; however, it is important to emphasize that other forms of tobacco should be addressed in parallel with the ultimate goal of reducing or eliminating all forms of tobacco consumption. This manuscript describes (1) examples at C3I sites to 
illustrate how involving patients' support persons could be critical in supporting them to be tobacco-free, (2) current practices and challenges among C3I cancer centers that include patients' support persons in their programs, and (3) a proposed research agenda to facilitate support persons' involvement in tobacco treatment in cancer centers.

\section{Real-Life Case Examples of Patients Served at C3I Cancer Centers}

Support persons of patients with cancer, both users or nonusers of tobacco, could be critical in patients' cancer care and in supporting them to be tobacco-free.

\section{Case 1: A Dual-Smoker Couple}

A female patient undergoing treatment for breast cancer reported difficulty in quitting combustible cigarettes because she and her husband smoked together. The tobacco cessation treatment enhanced the patient's cessation efforts by decreasing exposure to triggers and secondhand smoke, and assisted the husband to quit smoking. Both the patient and her husband were active participants in their synergistic cessation process.

\section{Case 2: A Patient With Cancer Who Hid His Smoking From His Family}

A male patient with nasopharyngeal cancer did not disclose his relapse of smoking to his family. The family members felt betrayed upon learning of his smoking status. With the patient's permission, the tobacco treatment specialist involved the family in his smoking cessation treatment. The treatment helped the patient manage the impact of smoking on his family relationships and facilitated family support for his cessation and relapse prevention efforts.

\section{Case 3: Needing Support to Quit Smoking}

A female patient undergoing treatment for anal cancer reported great difficulties in stopping smoking because her father, with whom she lived, smoked daily. The tobacco treatment specialist spoke with the father and offered smoking cessation resources. The father started individual smoking cessation counseling, and the patient attended weekly smoking cessation classes to support her effort to quit smoking.

\section{Current Practice of C3I Sites and Challenges}

As part of annual reporting, 43 of the C3I cancer centers responded to questions about involving patients' support persons in their tobacco treatment programs between July and December 2020. In that survey, fewer than half routinely addressed support persons in any of their tobacco cessation treatment practices $(46.5 \%$; $n=20)$ or assessed secondhand smoke exposure $(41.9 \% ; n=18)$. Only $20.9 \%(n=9)$ of programs asked support persons of patients who smoked if they would like to participate in the patient's tobacco use treatment plan. For patients' support persons who use tobacco, $32.6 \%(n=14)$ of programs offered resources (eg, educational pamphlets) and $25.6 \%(n=11)$ offered tobacco use treatment.

To obtain additional perspective related to engaging support persons in tobacco cessation treatment, we conducted a C3I Family and Social Support Systems Working Group Survey. Representatives of the 11 cancer centers participating in the Working Group cited a lack of protocols and difficulty in contacting support persons as the greatest challenges for their tobacco treatment program to engage support persons. Specifically, most of the centers cited a lack of protocols to (1) systematically identify support persons who use tobacco, (2) involve support persons in patients' tobacco treatment, (3) refer patients' support persons who use tobacco to cessation treatment, (4) intervene to reduce secondhand smoke exposure to patients, and (5) bill for activities involving patients' support persons. Table 1 shows strategies implemented at 3 C3I cancer centers to address these barriers, which include outreach efforts in partnership with the Community Outreach and Engagement team of cancer centers, elements of tobacco treatment program intake protocol, and a comprehensive tobacco treatment program to engage patients and their support persons.

\section{Proposed Research Agenda}

Engaging patients' support persons in efforts to improve cancer care and tobacco cessation outcomes remains unexplored. To address these gaps, the Working Group research agenda aims to establish (1) evidence-based strategies for engaging support persons in patients' cessation efforts, (2) interventions to provide cessation treatment options to patients' support persons, and (3) best practices to routinely identify and engage family and social support systems in patients' cessation efforts.

\section{Evidence-Based Strategies for Engaging}

Patients' Support Persons

Little is known about effective approaches to engage support persons of patients with cancer ${ }^{13,14}$ or smokers from the general population.,15 Mixed methods research exploring support persons' tobacco treatment needs and preferences can help in designing effective strategies. Engagement strategies should consider the role relationship and smoking status of support persons, with thoughtful integration of cultural and family contexts.

Interventions to Provide Cessation Treatment Options to Support Persons

Further research is needed to tailor tobacco treatment interventions to the specific needs of support persons in the context of the cancer care continuum, with special attention to cultural and social structural needs. Research 


\begin{tabular}{|c|c|c|c|}
\hline & $\begin{array}{l}\text { Dartmouth-Hitchcock } \\
\text { Norris Cotton } \\
\text { Cancer Center (NCCC) }\end{array}$ & $\begin{array}{l}\text { Memorial Sloan Kettering } \\
\text { Cancer Center (MSK) }\end{array}$ & $\begin{array}{l}\text { University of North Carolina } \\
\text { Lineberger Comprehensive } \\
\text { Cancer Center (UNC LCCC) }\end{array}$ \\
\hline $\begin{array}{l}\text { Geographic Location/ } \\
\text { Catchment Area }\end{array}$ & $\begin{array}{l}\text { Rural New Hampshire } \\
\text { Bistate catchment area } \\
\text { (New Hampshire and Vermont) }\end{array}$ & Metropolitan New York City & Across North Carolina \\
\hline $\begin{array}{l}\text { Example Activities to Address } \\
\text { Barriers and Engage Family and } \\
\text { Social Support Persons in } \\
\text { Tobacco Cessation Treatment }\end{array}$ & $\begin{array}{l}\text { - Collaborates with the } \\
\text { NCCC Community Outreach } \\
\text { and Engagement (COE) team } \\
\text { to increase the visibility of } \\
\text { tobacco treatment via in-person } \\
\text { and virtual outreach events } \\
\text { - Developed a game to educate } \\
\text { >4,500 attendees at NCCC's } \\
\text { annual fundraising event about } \\
\text { emerging tobacco product } \\
\text { concerns; Tobacco Treatment } \\
\text { Specialists spoke about TTP } \\
\text { services } \\
\text { Organized a resource fair for the } \\
\text { Great American Smokeout, } \\
\text { inviting representatives from } \\
\text { American Cancer Society, state } \\
\text { quitlines, and hospital employee } \\
\text { wellness program to speak } \\
\text { about tobacco use and their } \\
\text { tobacco treatment services }\end{array}$ & $\begin{array}{l}\text { Administers MSK TTP intake form } \\
\text { that includes questions to identify } \\
\text { and engage support persons in } \\
\text { TTP, including: } \\
\text { - What are your main triggers for } \\
\text { smoking (includes a response } \\
\text { option for other smokers or } \\
\text { tobacco users)? } \\
\text { - Please indicate family members } \\
\text { who regularly smoke or use } \\
\text { other tobacco products } \\
\text { - Please indicate whether you have } \\
\text { a smoke-free home and/or car } \\
\text { - Many of my family members, } \\
\text { friends and/or coworkers } \\
\text { smoke and/or use tobacco } \\
\text { products (0 [not at all] to } 10 \\
\text { [extremely true]) }\end{array}$ & $\begin{array}{l}\text { - The UNC Quit Together (QT) } \\
\text { program provides free, } \\
\text { individualized tobacco } \\
\text { cessation treatment to patients } \\
\text { and their support persons who } \\
\text { smoke. Developed a support } \\
\text { person recruitment toolkit, } \\
\text { including: } \\
\text { Workflow outlining steps for } \\
\text { recruiting and enrolling } \\
\text { support persons } \\
\text { Promotional flyer, program } \\
\text { information and consent } \\
\text { document } \\
\text { Introduction email to patient } \\
\text { with a request to forward to } \\
\text { their support persons } \\
\text { Introduction email to support } \\
\text { person with request to } \\
\text { forward to the patient (when } \\
\text { support person is first to } \\
\text { enroll in QT) }\end{array}$ \\
\hline $\begin{array}{l}\text { Targeted Activities to Address } \\
\text { Patients' Support Persons in } \\
\text { Tobacco Cessation Treatment }\end{array}$ & $\begin{array}{ll}\checkmark & \text { Offer/Refer resources } \\
\checkmark & \text { Intervene to reduce } \\
\text { secondhand smoke exposure }\end{array}$ & $\begin{array}{l}\checkmark \text { Identify support persons who } \\
\text { use tobacco } \\
\checkmark \text { Offer/Refer resources } \\
\checkmark \text { Involve support persons in } \\
\text { patient's TTP } \\
\checkmark \begin{array}{l}\text { Assess secondhand smoke } \\
\text { exposure }\end{array}\end{array}$ & $\begin{array}{ll}\checkmark & \text { Identify support persons who } \\
\text { use tobacco } \\
\checkmark \text { Offer/Refer resources } \\
\checkmark \text { Involve support persons in } \\
\text { patient's TTP } \\
\checkmark \quad \text { Provide treatment to support } \\
\text { persons who smoke }\end{array}$ \\
\hline
\end{tabular}

Abbreviation: TTP, tobacco treatment program.

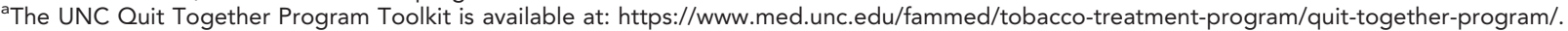

questions that examine the optimal frequency, duration, content, and format of tobacco interventions for support persons are needed. Adaptive clinical trial designs incorporating multiple modes of treatment delivery (telehealth, quitlines, in-person, and/or digital health applications) and biomarkers for secondhand smoke exposure assessment are warranted.

\section{Best Practices to Routinely Identify and Engage}

\section{Patients' Family and Social Support Systems}

To identify and engage support persons in tobacco treatment, new or refined clinical protocols and workflows are needed. Streamlining of the electronic health record to enroll support persons is important. Enhancements of the electronic health record will be pivotal to facilitate routine documentation and inclusion of support persons in patients' tobacco cessation efforts and cancer care. ${ }^{16}$

\section{Conclusions}

Involving support persons is critical to achieving goals of both cancer and tobacco treatment for patients with cancer who smoke. Promising interventions should be guided by evidence-based practices in tobacco treatment as well as caregiver support. The C3I Consortium, including the Family and Social Support Systems Working Group, offers a unique collaborative platform to advance these research efforts. The ultimate goal is to establish evidence-based practices to effectively engage family and social support systems of patients with cancer so that they can become tobacco-free.

\section{Acknowledgments}

The authors wish to thank the Tobacco Treatment Specialists at the University of North Carolina Tobacco Treatment Program and Suanne Harris, RN, CTTS, NCTTP, Co-Director of the Fontana Tobacco Treatment Center at the University of California San Francisco, for providing the deidentified case examples.

Cancer Center Cessation Initiative Family and Social Support Systems Working Group Members

Tracy E. Crane, PhD, RDN ${ }^{1, *}$; Janice Y. Tsoh, PhD ${ }^{2, *}$; Sarah D. Hohl, PhD, $\mathrm{MPH}^{3,+}$; Adam O. Goldstein, MD, MPH ${ }^{4,+}$; Katie L. Lenhoff, $\mathrm{MPH}^{5,+}$; Jamie Ostroff, $\mathrm{PhD}^{6,+}$; Kimberly Shoenbill, MD, $\mathrm{PhD}^{4,+} ;$ Kendall Fugate-Laus, $\mathrm{BS}^{7, \pm}$; Elyse R. Park, PhD, MPH ${ }^{8, \neq}$; Jennifer Peregoy, NCTTP ${ }^{9, \pm}$; Graham W. Warren, 
MD, $\mathrm{PhD}^{10, \pm}$; Robert Adsit, MEd ${ }^{11, \S}$; Mara Minion, $\mathrm{MA}^{3,5}$; Danielle Pauk, $\mathrm{BS}^{3, \text { s. }}$ and Betsy Rolland, PhD, MLIS, MPH ${ }^{3,12, \S}$

${ }^{1}$ Biobehavioral Health Sciences Division, College of Nursing, The University of Arizona, Tucson, Arizona; ${ }^{2}$ Department of Psychiatry and Behavioral Sciences, Helen Diller Family Comprehensive Cancer Center, University of California San Francisco, San Francisco, California; ${ }^{3}$ Carbone Cancer Center, School of Medicine and Public Health, University of Wisconsin-Madison, Madison, Wisconsin; ${ }^{4}$ Department of Family Medicine, University of North Carolina, Chapel Hill, North Carolina; ${ }^{5}$ Norris Cotton Cancer Center, DartmouthHitchcock Medical Center, Dartmouth, New Hampshire; ${ }^{6}$ Department of Psychiatry and Behavioral Sciences, Memorial Sloan Kettering Cancer Center, New York, New York; ${ }^{7}$ Department of Psychology, Virginia Commonwealth University, Richmond, Virginia; ${ }^{8}$ Department of Psychiatry, Massachusetts General Hospital, Boston, Massachusetts; ${ }^{9}$ Cancer Center, University of Virginia, Charlottesville, Virginia; ${ }^{10}$ Department of Radiation Oncology, Medical University of South Carolina, Charleston, South Carolina; ${ }^{11}$ Department of Medicine, Center for Tobacco Research and Intervention, School of Medicine and Public Health, University of Wisconsin-Madison; and ${ }^{12}$ Institute for Clinical and Translational Research, University of Wisconsin-Madison, Madison, Wisconsin.

"Working Group Chair; ${ }^{+}$Writing Group Author; ${ }^{*}$ Working Group Author ${ }^{\S}$ Coordinating Center Author.

Submitted July 26, 2021; final revision received September 14, 2021 accepted for publication September 16, 2021

Disclosures: G.W. Warren has disclosed participating in research for the Agency for Healthcare Research and Quality and serving as a consultant for the Canadian Partnership Against Cancer. The remaining individuals have disclosed no relevant financial relationships.

Funding: This supplement was funded by the $\mathrm{C} 3 \mathrm{l}$ Coordinating Center contract from the National Cancer Institute (CRDF Award \#66590). In addition, authors received funding for their $\mathrm{C} 3 \mathrm{l}$ participation via a supplement to their NCl P30 cancer center support grant during the period 2017-2021.

Correspondence: Janice Y. Tsoh, PhD, University of California San Francisco, 401 Parnassus Avenue, San Francisco, CA 94143. Email: janice.tsoh@ucsf.edu

\section{References}

1. Kim J, Han JY, Shaw B, et al. The roles of social support and coping strategies in predicting breast cancer patients' emotional well-being: testing mediation and moderation models. J Health Psychol 2010;15:543552 .

2. Aprilianto E, Lumadi SA, Handian FI. Family social support and the selfesteem of breast cancer patients undergoing neoadjuvant chemotherapy. J Public Health Res 2021;10.

3. Hubbard G, Gorely T, Ozakinci G, et al. A systematic review and narrative summary of family-based smoking cessation interventions to help adults quit smoking. BMC Fam Pract 2016;17:73

4. Berg CJ, Thomas AN, Mertens AC, et al. Correlates of continued smoking versus cessation among survivors of smoking-related cancers. Psychooncology 2013;22:799-806.

5. Ruebush E, Mitra S, Meyer C, et al. Using a family systems approach to treat tobacco use among cancer patients. Int J Environ Res Public Health 2020;17:2050

6. Simmons VN, Sutton SK, Meltzer LR, et al. Preventing smoking relapse in patients with cancer: a randomized controlled trial. Cancer 2020;126: 5165-5172.

7. Hopenhayn C, Christian WJ, Christian A, et al. Factors associated with smoking abstinence after diagnosis of early stage lung cancer. Lung Cancer 2013:80:55-61.

8. Eng L, Su J, Qiu X, et al. Second-hand smoke as a predictor of smoking cessation among lung cancer survivors. J Clin Oncol 2014;32:564-570.
9. Eng L, Qiu X, Su J, et al. The role of second-hand smoke exposure on smoking cessation in non-tobacco-related cancers. Cancer 2015;121: 2655-2663.

10. Manning M, Wojda M, Hamel L, et al. Understanding the role of family dynamics, perceived norms, and lung cancer worry in predicting secondhand smoke avoidance among high-risk lung cancer families. J Health Psychol 2017;22:1493-1509.

11. Bottorff JL, Robinson CA, Sarbit G, et al. A motivational, gender-sensitive smoking cessation resource for family members of patients with lung cancer. Oncol Nurs Forum 2015;42:363-370.

12. Croyle RT, Morgan GD, Fiore MC. Addressing a core gap in cancer care the $\mathrm{NCl}$ Moonshot Program to help oncology patients stop smoking. N Engl J Med 2019;380:512-515.

13. Bastian LA, Fish LJ, Peterson BL, et al. Proactive recruitment of cancer patients' social networks into a smoking cessation trial. Contemp Clin Trials 2011;32:498-504

14. McDonnell KK, Hollen PJ, Heath J, et al. Recruiting family dyads facing thoracic cancer surgery: Challenges and lessons learned from a smoking cessation intervention. Eur J Oncol Nurs 2016:20:199-206.

15. Faseru B, Richter KP, Scheuermann TS, et al. Enhancing partner support to improve smoking cessation. Cochrane Database Syst Rev 2018;8: CD002928.

16. Applebaum AJ, Kent EE, Lichtenthal WG. Documentation of caregivers as a standard of care. J Clin Oncol 2021:39:1955-1958. 